\title{
Miranda
}

Revue pluridisciplinaire du monde anglophone /

Multidisciplinary peer-reviewed journal on the English-

speaking world

$11 \mid 2015$

Expressions of Environment in Euroamerican Culture / Antique Bodies in Nineteenth Century British

Literature and Culture

\section{Sandrine Sorlin, La Stylistique anglaise. Théories et pratiques}

Nathalie Vincent-Arnaud

\section{OpenEdition}

Journals

Édition électronique

URL : https://journals.openedition.org/miranda/7125

DOI : 10.4000/miranda. 7125

ISSN : 2108-6559

Éditeur

Université Toulouse - Jean Jaurès

\section{Référence électronique}

Nathalie Vincent-Arnaud, «Sandrine Sorlin, La Stylistique anglaise. Théories et pratiques », Miranda [En ligne], 11 | 2015, mis en ligne le 22 juillet 2015, consulté le 08 septembre 2022. URL : http:// journals.openedition.org/miranda/7125; DOI : https://doi.org/10.4000/miranda.7125

Ce document a été généré automatiquement le 8 septembre 2022.

\section{cc)}

Creative Commons - Attribution - Pas d'Utilisation Commerciale - Pas de Modification 4.0 International - CC BY-NC-ND 4.0

https://creativecommons.org/licenses/by-nc-nd/4.0/ 


\title{
Sandrine Sorlin, La Stylistique anglaise. Théories et pratiques
}

\author{
Nathalie Vincent-Arnaud
}

\section{RÉFÉRENCE}

Sandrine Sorlin, La Stylistique anglaise. Théories et pratiques (Rennes : Presses

Universitaires de Rennes), 228 p, ISBN 978-2-7535-3446-9

1 La publication d'un nouvel ouvrage de stylistique dans le domaine de l'anglistique ne peut que réjouir tous ceux qui, depuis longtemps, font de cette discipline - qui est en réalité bien plus un carrefour d'outils et de pratiques qu'une discipline à proprement parler - une approche privilégiée du texte, ce dernier fût-il littéraire ou non (même si les textes non littéraires n'ont $\mathrm{eu}$, pendant très longtemps, qu'une place très marginale). Cette publication - entre autres intervenues ces dernières années - ne peut également que refléter la grande vitalité de la SSA (Société de Stylistique Anglaise) et l'enthousiasme de ses participants qui, au fil des colloques, des ateliers tenus chaque année au Congrès de la SAES, des numéros d'Études de Stylistique Anglaise (revue qui a succédé en 2011 au Bulletin de Société de Stylistique Anglaise), ne se dément pas. Ce dynamisme fait écho à celui qui paraît, depuis plusieurs années, envahir la scène littéraire dans son ensemble, toutes littératures et langues confondues, des écrits fondateurs de Georges Molinié (La Stylistique [Paris : Presses Universitaires de France, 1993]) à ceux de Laurent Jenny (Le Style en acte. Vers une pragmatique du style [Genève : éditions Métis, 2011]) en passant par les approches trans-sémiotiques, voire «pansémiotiques » d'Anne Herschberg-Pierrot (Le Style en mouvement : littérature et art [Paris : Belin, 2005]) et de Jean-Jacques Lecercle («La stylistique deleuzienne et les petites agrammaticalités », Bulletin de la Société de Stylistique Anglaise $n^{\circ} 30$ [Nanterre : Presses de l'Université de Nanterre, 2008, 273-286; "Pour une stylistique des singularités », Bulletin de la Société de Stylistique Anglaise $\mathrm{n}^{\circ} 31$ [Nanterre, Presses de l'Université de Nanterre, 2008, 21-32]). Entre autres témoignages récents de cet intérêt renouvelé englobant le champ de l'anglistique mais dépassant largement ses frontières 
figure l'ouvrage dirigé par Cécile Narjoux, Au-delà des frontières: perspectives de la stylistique contemporaine (Frankfurt: Peter Lang, 2012), rassemblant des contributions issues d'un colloque tenu à Dijon en 2011 sur le thème «Nouvelles stylistiques: nouvelles approches linguistiques du texte littéraire?» et auquel ont participé plusieurs stylisticiens anglicistes.

2 Sandrine Sorlin, membre de la SSA et auteur du présent ouvrage, pratique une stylistique résolument ouverte (voir Sandrine Sorlin, La Défamiliarisation linguistique dans le roman anglais contemporain [Montpellier: Presses Universitaires de la Méditerranée, coll. « Horizons Anglophones », 2010]; Sandrine Sorlin, Langue et autorité: de l'ordre linguistique à la force dialogique [Rennes: Presses Universitaires de Rennes, coll. «Rivages linguistiques », 2012]), adjectif qui pourra paraître redondant aux uns, praticiens convaincus, mais quelque peu surprenant aux autres, qui auraient exclusivement à l'esprit le modèle stéréotypé et pour le moins réducteur de l'ancienne rhétorique. S'il n'est évidemment pas question de méconnaître l'héritage de celle-ci, la stylistique n'en fait pas moins l'objet, depuis une vingtaine d'années, d'un «salutaire retour réflexif et épistémologique " (Editorial de la revue électronique de l'Association Internationale de Stylistique, "Le style en questions ", n 135/136, décembre 2007) de l'aveu même de ceux qui la pratiquent et n'en sont peut-être venus à la pratiquer que grâce en partie à ce renouvellement (dont ils ont parfois été les initiateurs). Cette stylistique en pleine mutation accorde notamment une place de choix aux outils de la linguistique, d'une linguistique foncièrement énonciative, abondamment nourrie des apports mentalistes et pragmatiques, puis redéployée et ré-ensemencée dans le champ de la philosophie du langage à la faveur, notamment, de l'essor des théories cognitives. "[E]ntre rhétorique et linguistique ", comme la définissait en 2002 un numéro de la revue Langue française, telle est la nature de ce carrefour déjà évoqué où elle se situe. Cette définition par défaut ne fait qu'appeler et confirmer l'existence de nombreux possibles, d'ajouts et consolidations incessants à apporter à l'édifice conceptuel et méthodologique, d'autant de matériaux qui, jusqu'à présent, ont forgé un amalgame souvent efficace pour ce qui demeure avant tout une "technique de lecture et d'interprétation " (Laurent Jenny, Le Style en acte. Vers une pragmatique du style [Genève : éditions Métis, 2011, 100]). Corrélativement, le statut d'entre-deux de la stylistique lui confère sa mutabilité, son adaptabilité, son caractère essentiellement protéiforme qui lui fait « user et changer les outils» (Georges Molinié, Eléments de stylistique française [Paris : Presses Universitaires de France, 1986, 140]) qui la constituent en fonction de la nature même de l'objet qu'elle embrasse. Elle pourrait ainsi se définir comme l'assurance d'un jugement esthétique non figé dans des grilles de fonctionnement préétablies, à l'image du discours et de l'écriture eux-mêmes; comme la garantie de ce «flux » de pensée dont Laurent Jenny fait une "arme contre la solidité des choses » (Laurent Jenny, La Vie esthétique. Stases et flux [Lagrasse: Verdier, 2013, 54]) dans sa réflexion sur la musique et sur l'accès, par son entremise, à une forme de «lointain intérieur ». Ce dernier n'est autre que l'objet même de la quête du stylisticien, tout à l'écoute, pour ce faire, de la petite musique d'un texte et de sa manière de résonner en nous.

3 C'est sur cette mobilité de la pensée critique, sur cette appréciation esthétique en mouvement car susceptible de recueillir les fruits d'une véritable transversalité épistémologique, que s'appuie l'ouvrage de Sandrine Sorlin. Sa construction est le reflet de ce fondement, faisant table rase des divisions observables dans de nombreux 
manuels de stylistique antérieurs, souvent fournies par les figures de style ou autres catégories linguistiques proposées comme point de départ de l'étude d'un texte (ex. : le nom / le verbe / la syntaxe). Ce qui est revendiqué ici, à travers l'organisation même de l'ouvrage, est un décloisonnement de ces catégories, propice à l'instauration d'un dialogue entre, d'une part, outils et questionnements plus traditionnels (les outils de la narratologie et de l'énonciation, par exemple, et les questions de genre et de registre) et, d'autre part, perspectives plus novatrices : au nombre de ces dernières, qui rendent la lecture de l'ouvrage particulièrement stimulante, figurent la contribution des travaux de Monique de Mattia-Viviès à l'étude des avatars du discours indirect libre et du monologue intérieur (Monique de Mattia-Viviès, Le Discours indirect libre au risque de la grammaire [Aix-en-Provence: Publications de l'Université de Provence, 2006]) les apports fournis par Leech, Grice, Brown et Levinson depuis les années 1980 à la pragmatique et aux interactions verbales (avec notamment la notion de Face Threatening Acts et celle, complémentaire, d' «impolitesse linguistique »; voir Denis Jamet et Manuel Jobert (dir.), Empreintes de l'euphémisme: tours et détours [Paris: L'Harmattan, 2010] et Denis Jamet et Manuel Jobert (dir.), Aspects of Linguistic Impoliteness. Newcastle: Cambridge Scholars, 2013), mais aussi, de manière extrêmement nourrie, le "tournant cognitif » de la stylistique. Ce dernier, qui fait l'objet du dernier chapitre d'une trentaine de pages, est abordé à travers les théories complémentaires des «cadres contextuels» et des «mondes textuels» auxquelles se rattache une réflexion sur les "schémas" émotionnels et perceptifs engrangés mentalement par l'expérience grâce auxquels "nous serions capables de faire sens d'expériences nouvelles similaires" (181) et à "percevoir les mots sous un jour nouveau » (182). Mises à l'honneur, comme le rappelle l'auteur, dans les travaux de George Lakoff mais aussi dans ceux, plus récents, de Peter Stockwell, les figures de la métaphore et de la métonymie sont revisitées à la lumière du rôle structurant qu'elles comportent pour l'imaginaire à l'œuvre dans un texte, voire dans un ensemble de textes auxquels elles assignent des traits génériques: les exemples donnés en fin de parcours sont ceux de l'utopie et de la dystopie, cette dernière étant évoquée par l'auteur, dans la mouvance de Stockwell, comme entretenant avec la réalité une relation "d'ordre métonymique » car elle "relève en effet davantage de la caricature déformée de notre réalité qu'elle ne crée un monde alternatif » (200).

Outre la profondeur de la réflexion proposée et une hauteur de vue qui n'a d'égale que la fluidité et la clarté pédagogique de l'ensemble, on apprécie le déplacement constant du regard de l'auteur de l'angle panoramique aux "faits de texture » (Jean-Michel Adam, in Molinié, Georges et Pierre Cahné (dir.), Qu'est-ce que le style? [Paris : Presses Universitaires de France, 1994, 19]) les plus ténus mais pouvant enserrer tout un réseau de sens à déployer, tels les effets parodiques et le jeu des voix dans un poème inédit de Michael Kindellan, "Widespread Losses» (91-4). Le corpus utilisé, majoritairement littéraire, affiche une certaine prédilection de l'auteur pour les textes des $20^{\text {ème }}$ et $21^{\text {ème }}$ siècles, même si Shakespeare, Fielding, Sterne ou Hawthorne ne manquent pas à l'appel. Cette inclination pour le contemporain se fait jour également dans les quelques extraits de journaux divers, du New Yorker au Guardian, qui fournissent des exemples fort utiles de la structuration de l'information dans le discours (et illustratifs de l'ouverture de la stylistique au champ non littéraire). Hormis le plaisir de la nouveauté qu'il comporte pour le lecteur, ce choix contribue à la cohérence d'ensemble tout en dévoilant aussi bien la diversité que les matériaux communs des chemins expressifs dans lesquels une société, une époque, un genre 
littéraire se sont engagés. On pourrait aisément imaginer, dans ce domaine, de féconds points de rencontre entre, d'une part, de nombreux aspects développés par Sandrine Sorlin quant à la construction des imaginaires et, d'autre part, la «stylistique des imaginaires langagiers » et des « patrons stylistiques» proposée par Julien Piat (« Vers une stylistique des imaginaires langagiers ", Corpus 5 : 2006, 113-141).

La démarche d'ensemble s'est de toute évidence fondée sur ce que l'auteur a appelé Inventive Linguistics, titre d'un ouvrage qu'elle a dirigé suite à un colloque tenu à Montpellier sur ce même thème, et, corrélativement, sur cette « défamiliarisation » qui donne également son titre à un autre de ses ouvrages, dans l'optique du "reste » et de la "stylistique des singularités " chère à Jean-Jacques Lecercle. Défamiliarisation, ouverture, créativité, confrontation avec l'inattendu apparaissent bel et bien comme les maitres mots du présent ouvrage : dans la nature des textes eux-mêmes et dans la méthode employée pour leur analyse, dans la conclusion en forme d'ouvertures toujours plus poussées sur la diversité des champs et des modes d'exploration qui s'offrent au chercheur, mais aussi, au fil des pages qui précèdent, dans l'appel constant qui est lancé au lecteur. Il en est ainsi des petits encadrés fréquents qui invitent, via des consignes claires et attrayantes, à une forme de creative writing, d'exercices de style par le biais de la rédaction d'un petit texte censé faciliter l'accès aux divers cheminements mentaux qui sous-tendent l'écriture. Ce contrat dialogique, qui fait entrer le lecteur dans ce même processus créatif qu'il doit apprécier, est d'emblée précisé par l'auteur : Le présent ouvrage n'est pas une grammaire mais il propose une exploitation possible de la grammaire à travers deux activités qui sont souvent pratiquées séparément : la lecture et l'écriture. [...] Associer une pratique d'écriture à l'analyse permet de s'assurer des marqueurs commentés et de la compréhension de l'ensemble des paramètres qui donnent sens etforme au Texte. (49-50)

Espérons d'abord que ce livre, à la fois érudit et foncièrement ouvert et novateur, créatif et récréatif, porteur d'un authentique enthousiasme à partager et à cultiver, réjouira tous ceux que le pouvoir du verbe fascine, enchante, étonne, inquiète, déstabilise de multiples manières. Espérons aussi qu'il convaincra de la nécessité de décloisonner les outils et les méthodes pour tenter de saisir le langage et l'esprit humains dans leur complexité, celle de tous les possibles, advenus ou à venir, dans une littérature entendue au sens très large et renouvelable à l'infini, produite par cette " espèce fabulatrice » (Nancy Huston, L'Espèce fabulatrice [Arles : Actes Sud, 2008]) dont l'essence est de (se) raconter et de (se) ré-inventer.

\section{INDEX}

Keywords : cognition, enunciation, linguistics, pragmatics, stylistics

Mots-clés : cognition, énonciation, linguistique, pragmatique, stylistique 


\section{AUTEURS}

\section{NATHALIE VINCENT-ARNAUD}

Professeur

Université Toulouse-Jean Jaurès

nathalie.vincentarnaud@sfr.fr 